\title{
COMPARISON OF DELAYED CORD CLAMPING AND CORD MILKING EFFECTS ON NEONATAL APGAR SCORE AND HAEMATOCRIT
}

\author{
Ghulam Sabir, Ayesha Raja*, Saira Mahboob**, Saima Shoukat*, Ali Kashif**, Shazia Iffat*** \\ Combined Military Hospital Hyderabad/National University of Medical Sciences (NUMS) Pakistan, ${ }^{*}$ Combined Military Hospital Malir/National University of \\ Medical Sciences (NUMS) Pakistan, ${ }^{* *}$ Pakistan Naval Ship Shifa Hospital, Karachi Pakistan, ${ }^{* * *}$ Combined Military Hospital Attock/National University of \\ Medical Sciences (NUMS) Pakistan
}

\section{ABSTRACT}

Objective: To assess the feasibility of umbilical cord milking as an alternative to delayed cord clamping during caserean section by comparing neonatal outcomes.

Study Design: Quasi experimental study.

Place and Duration of Study: Department of Anesthesiology, Combined Military Hospital, Okara, from Mar to Oct 2018. Methodology: A total of 384 cases (n=192 in each group) were included in our study. In group A, the half-length of umbilical cord was milked thrice by the operating surgeon before clamping and cutting it. In group B the umbilical cord was cut 90 second after delivery of the baby. The outcomes were: APGAR score at one and five minutes after delivery, haemglobin and haematocrit 4 hours after delivery of baby. Requirement of oxygen and ventilatory support during the 24 hours after birth were also noted.

Results: Our two study groups didn't vary in their demographic profile. The difference between the APGAR score at one and five minutes was not statistically significant, $(p>0.05)$. The diffrence in haemoglobin and haematocrit levels of the neonates 4 hours after delivery were insignificant ( $p$-value 0.27 and 0.14 respectively).

Conclusion: Milking of the umbilical cord can be used as an alternative to the recommended technique of delayed umbilical cord clamping, especially when immediate resuscitation of the newborn is needed.

Keywords: APGAR score, Caesarean section, Delayed cord clamping, Neonatal haematocrit, Umbilical cord milking.

How to Cite This Article: Sabir G, Raja A, Mahboob S, Shoukat S, Kashif A, Iffat S. Comparison of Delayed Cord Clamping and Cord Milking Effects on Neonatal Apgar Score and Haematocrit. Pak Armed Forces Med J 2021; 71(5): 1811-1814. ～doi: https://doi.org/10.51253/pafmj.v71i5.3508

\footnotetext{
This is an Open Access article distributed under the terms of the Creative Commons Attribution License (https://creativecommons.org/licenses/by-nc/4.0/), which permits unrestricted use, distribution, and reproduction in any medium, provided the original work is properly cited.
}

\section{INTRODUCTION}

In the recent past the rate of caesarean delivery has considerablly increased with documented negative health effects. ${ }^{1}$ Although latest guidelines recommends delayed cord (more than 1 minutes) after delivery of term and preterm neonate, but in intrauterine hypoxia and fetal distress, the recommendation is to omit the delayed cord clamping and start resuscitation as well as positive pressure ventilation without delay. ${ }^{2}$ However mostly the cord is immediately cut during third stage of labor and caesarean delivery. A study by Boere et al reported the practice of early cord clamping in $81 \%$ of cesarean section versus $6 \%$ in uncomplicated vaginal deliveries. ${ }^{3}$ The reason for immediate cutting of umbilical cord can be, urge to handing over the baby to neonatal resuscitation team or unintended haste for the management of third stage of labour. ${ }^{4}$ There is no evidence that cutting the cord immedialey after delivery is beneficial for the parturient or the new born. Cord clamping immediately after birth is potentially

Correspondence: Dr Ghulam Sabir, Classified Anaesthesiologist, Combined Military Hospital, Hyderabad Pakistan

Received: 20 Nov 2019; revision received: 13 Feb 2020; accepted: 19 Feb 2020 harmful in a new born with compromised cardiovascular and respiratory functions. 5,6

In Europe 26\% and in low income countries 100\%, children of preschool going age group suffer from Iron deficiency. ${ }^{7}$ Iron deficiency causes anaemia and in early childhood can cause irreversible adverse effects on neurodevelopment. Supplemental iron in children on risk of iron deficiency results in improved psychomotor and cognitive development. ${ }^{8}$ If clamping of the umbilical cord is delayed after delivery of baby, additional blood will be provided by autotransfusion to the newborn and consequently will improve the iron status. Delayed cord clamping is one of the measures that can prevent iron deficiency in early childhood. The milking of the cord by grasping the unclamped umbilical cord and pushing the blood towards the neonate can autotransfuse placental blood to neonate much faster as compared to waiting for 2-3 minutes in delayed cord clamping. ${ }^{9}$ However this rapid transfusion may cause circulatory overload requiring treatment including ventilatory support and supplemental oxygen. The study in preterm neonates is reported to be devoid of such side effects. ${ }^{10}$ 


\section{Neonatal Apgar Score And Haematocrit}

According to authors' knowledge, limited data is available in Asian population regarding use on umblical cord milking in term neonates delivered by cesarean section. The objective of this study was to assess the feasibility of umblical cord milking as an alternative to delayed cord clamping during cesarean section by comparing neonatal outcomes. If cord milking proved comparable to delayed cord clamping, then it can be used as alternative to standard method in intrauterine compromised neonates and will avoid unnecessary delay in resuscitation of the new born.

\section{METHODOLOGY}

After getting approval from the hospital ethics committee (IERC/Anes/2018/03), the study was conducted at Combined Military Hospital, Okara Pakistan, from March to October, 2018. WHO sample size calculator was used to calculate sample size. The total sample size of study was 384 (192 in each group) by keeping level of confidence measure 1.96, margin of error 0.05 , and the power of test $80 \%$ and design effect 1 . Consecutive non probability was used for sampling. A total of 384 patients ( $\mathrm{n}=192$ in each group) undergoing elective caesarean under spinal anaesthesia were included in this randomized clinical trial.

Inclusion Criteria: Parturient with gestational age $\geq 37$ weeks and singleton pregnancy were included in this study. To rule out heterogeneity; no limit of mother's age, weight or height was kept for inclusion of in this study.

Exclusion Criteria: Parturient with rhesus negative blood group, maternal ASA physical class status $\geq \mathrm{III}$, fetal congenital anomalies and intrauterine growth retardation were excluded. All the patients who refused spinal anesthesia were also excluded from study.

The pregnant ladies were assessed and prepared for surgery as per institute protocol, after counselling and informed consent, they were kept nothing per oral at least 6 hours pre-operatively and started with intravenous fluids and antibiotics. On arrival in operation theatre the patient profile like name, ASA physical status of mother, preoperative haemoglobin of the mother and gestational age of the baby were recorded. The parturants were allotted either the umbilical cord milking group (A) or delayed cord clamping group (B) by lottery method. Neonatal resuscitation team and the staff working in Nursery were kept unaware of the grouping. At the time of surgery ASA standard I and II monitoring was started. Intra venous access was ensured by 18 gauge cannula. Premedication and technique for spinal anesthesia were same in both the groups.
Dexamethasone $8 \mathrm{mg}$ and $10 \mathrm{mg}$ metoclopramide were given as premedication. Spinal anaesthesia was given in sitting position after subcutaneous infiltration of 4 milliliter of $1 \%$ xylocain at intervetebral level Lv3-4 or Lv4-5. Twenty seven gauge quinke bebcock spinal needles were used in all patients to locate the intrathecal space. About $11.25 \mathrm{mg}$ of $0.75 \%$ bupivacain heavy was given intrathecally after achieving the free flow of cerebrospinal fluid. Then parturants were placed in supine position with left uterine displacement. The effect of spinal anesthesia was assessed using Bromage score and pin prick method at 5 and 10 minutes. Surgical incision was given, after confirming adequacy of surgical anesthesia till T6 level.

The time of delivery was noted on proforma as soon as baby was delivered. In group A, after delivery, neoborn was placed about $15 \mathrm{~cm}$ below the level of the palcenta on side of mother. The operating obsteterician grasped the umbilical cord of the neonate between thumb and index finger, at aproximate half length of umbilical cord. The umbilical cord was gently milked towards neonate. The umbilical cord was clamped and cut after milking thrice. In group B newborn was kept $15 \mathrm{~cm}$ below the level of placenta. The time keeper started the stopwatch as soon as the newborn was placed in the forementioned position and informed the operating obstetrician on completion of 90 seconds. The cord was clamped on completion of 90 seconds and cut immediately.

The baby was then handed over to neonatal resuscitation team. APGAR score of the new born was assessed at 1 minute and 5 minutes of resuscitation and was marked on the research proforma. After resuscitation baby was shifted to nursery with adjacent and interconnected Neonatal Intensive Care Unit (NICU). The sister in nursery collected the venous sample of the newborn on completion of 4 hours after delivery and dispatched to pathology laboratory for haemoglobin and haematocrit levels. Baby was monitored in NICU and supplemental oxygen or ventilatory supports were initiated as per standard protocol of NICU and was marked on the proforma. Proforma were collected after completion of 24. The haemoglobin and haematocrit were collected from the pathology laboratory and entered in the proforma.

SPSS-25 was used to analyze results. The demographic data was described as mean and standard. The numeric data were analyzed by using independent samples t-test. The categorical data were described as 
frequency and percentage; and chi-square test was applied. A $p$-value $\leq 0.05$ was taken as significant.

\section{RESULTS}

Total 384 newborns delivered by lower segment caesarean section under spinal anaesthesia were included in the study ( $\mathrm{n}=192$ in each group) The two groups didn't vary in terms of haemoglobin levels of the mothers and gestational age of the new born, their, $p$-value $>0.05$ (Table-I). The comparison of neonatal outcome in terms of APGAR score at one and five minutes, haemoglobin and haematocrit at four hours after birth are given in the Table-II.

Total 48 neonates from both the groups required supplemental oxygen while only 2 required ventilatory support in first 24 hours after birth. The difference between regarding oxygen requirement and ventilatory support in 24 hours after birth was not statistically significant. The details are given in the Table-III.

Table-I: Demographic profile of study groups.

\begin{tabular}{|c|c|c|c|c|c|c|}
\hline \multirow{2}{*}{\multicolumn{2}{|c|}{ Variables }} & \multirow{2}{*}{\multicolumn{2}{|c|}{$\begin{array}{c}\begin{array}{c}\text { Group A } \\
(\mathbf{n}=\mathbf{1 9 2})\end{array} \\
\text { Mean } \pm \text { SD }\end{array}$}} & \multirow{2}{*}{\multicolumn{2}{|c|}{$\begin{array}{c}\begin{array}{c}\text { Group B } \\
(\mathbf{n}=\mathbf{1 9 2})\end{array} \\
\text { Mean } \pm \text { SD }\end{array}$}} & \multirow{2}{*}{$\begin{array}{c}p- \\
\text { value }\end{array}$} \\
\hline & & & & & & \\
\hline \multicolumn{2}{|c|}{$\begin{array}{l}\text { Mother's haemoglobin } \\
(\mathrm{g} / \mathrm{dL})\end{array}$} & \multicolumn{2}{|c|}{$11.46 \pm 1.04$} & \multicolumn{2}{|c|}{$11.25 \pm 0.94$} & 0.11 \\
\hline \multicolumn{2}{|c|}{ Gestational age (weeks) } & $38.22 \pm 0.86$ & & \multicolumn{2}{|c|}{$38.65 \pm 0.91$} & 0.19 \\
\hline \multicolumn{7}{|c|}{$\begin{array}{l}\text { Table-II: Neonatal APGAR score, haemoglobin and } \\
\text { haematocrit. }\end{array}$} \\
\hline \multirow{2}{*}{\multicolumn{2}{|c|}{ Variables }} & \multicolumn{2}{|c|}{$\begin{array}{l}\text { Group A } \\
(n=192)\end{array}$} & \multicolumn{2}{|c|}{$\begin{array}{c}\text { Group B } \\
(\mathrm{n}=192)\end{array}$} & \multirow{2}{*}{$\begin{array}{c}p- \\
\text { value }\end{array}$} \\
\hline & & \multicolumn{2}{|c|}{ Mean \pm SD } & \multicolumn{2}{|c|}{ Mean \pm SD } & \\
\hline \multicolumn{2}{|c|}{ APGAR score at 1 minute } & \multicolumn{2}{|c|}{$7.58 \pm 0.564$} & \multicolumn{2}{|c|}{$7.59 \pm 0.592$} & 0.57 \\
\hline \multirow{2}{*}{\multicolumn{2}{|c|}{$\begin{array}{l}\text { APGAR score at } 5 \text { minute } \\
\text { Haemoglobin }(\mathrm{g} / \mathrm{dL})\end{array}$}} & \multicolumn{2}{|c|}{$9.69 \pm 0.667$} & \multicolumn{2}{|c|}{$9.65 \pm 0.578$} & 0.84 \\
\hline & & \multicolumn{2}{|c|}{$18.18 \pm 1.84$} & \multicolumn{2}{|c|}{$17.55 \pm 2.07$} & 0.27 \\
\hline \multicolumn{2}{|l|}{ Haematocit (\%) } & \multicolumn{2}{|c|}{$52.95 \pm 5.48$} & \multicolumn{2}{|c|}{$51.33 \pm 6.24$} & 0.14 \\
\hline \multicolumn{7}{|c|}{$\begin{array}{l}\text { Table-III: Requirement of supplemetary oxygen and } \\
\text { ventilatory support in } 24 \text { hours after birth. }\end{array}$} \\
\hline \multirow{2}{*}{ Variables } & \multicolumn{2}{|c|}{ Group A $(n=192)$} & \multicolumn{3}{|c|}{ Group B (n=192) } & \multirow{2}{*}{$\begin{array}{c}p \text { - } \\
\text { value }\end{array}$} \\
\hline & Yes (\%) & No (\%) & Yes & $(\%)$ & No $(\%)$ & \\
\hline $\begin{array}{l}\text { Oxygen supple- } \\
\text { mentatiom }\end{array}$ & $\begin{array}{c}25 \\
(13.02) \\
\end{array}$ & $\begin{array}{c}167 \\
(86.98) \\
\end{array}$ & & $.98)$ & $\begin{array}{c}169 \\
(88.02) \\
\end{array}$ & 0.439 \\
\hline $\begin{array}{l}\text { Ventialtory } \\
\text { support }\end{array}$ & $\begin{array}{c}1 \\
(0.52)\end{array}$ & $\begin{array}{c}191 \\
(99.48)\end{array}$ & & $\begin{array}{l}1 \\
52)\end{array}$ & $\begin{array}{c}191 \\
(99.48)\end{array}$ & 0.751 \\
\hline
\end{tabular}

\section{DISCUSSION}

In this study, the APGAR score of neonate at one and five minutes, haemoglobin and haematocrit of newborn 4 hours after delivery and requirement of oxygen and ventilatory support in first twenty four hours after delivery remained comparable in our two study groups ( $p$-value $>0.05$ ).

During the caesarean delivery usually umbilical cord is immediately clamped and cut in a haste to avoid hypothermia and h0.and over the baby to the resuscitation team. ${ }^{11}$ Delayed cord clamping results in transfusion of placental blood to the newborn and is recommended to improve iron stores in the early months of life but the ideal clamping time is still debateable. ${ }^{12-14}$ The placental transfusion occurs mainly in the first minute after birth and is completed in three minutes and this delay in cord clamping is devoid of any adverse effect for the newborn. ${ }^{15,16}$ This early cord clamping deprive the baby of transfer of blood from the placenta to baby. The studies have suggested that negative intrathoracic pressure during the initial few breaths significantly improves the placental transfusion. ${ }^{17}$ McDonald et al, concluded in Cochrane Database of systemic Reviews of 2013 that a delayed cord clamping of either more than one minute or when the umbilical cord stop pulsating is associated with higher haemglobin levels at birth and lower rate of iron deficiency anaemia in early childhood without any maternal complication or negative impact on her health. ${ }^{18} \mathrm{~A}$ study at Swedish country hospital, of Halland indicated that delayed cord clamping results in better scores of children in social and fine motor domains at their age of four years. ${ }^{19}$ The additional supply of blood during delayed cord clamping provides approximately 40$50 \mathrm{mg} / \mathrm{kg}$ iron to neonate which is sufficient enough to increase the ferritin levels in early childhood and prevents iron deficiency anaemia during the first year of life. ${ }^{20}$ Particularly in developing countries where iron deficiency is common, the delayed cord clamping provides enough iron till age at which other easy interventions can be done to improve it. ${ }^{21}$

Milking of the umbilical cord can increase placental transfusion to the newborn and a very short span of time will be required to achieve the required amount of transplacental transfusion to the newborn rather than waiting for the cord pulsation to stop. Only limited data is available which compared the delayed cord clamping with milking of the cord. Jaiswal et al, ${ }^{22}$ studied the effects of delayed cord clamping and umbilical cord milking in term infants, they found no significance difference in haemoglobin levels at 30 minutes after birth ( $p$-value 0.37), 48 hours after birth ( $p$-value $=0.41)$ and at 6 weeks of age ( $p$-value $=0.50)$. This study also showed no significant difference between two study groups in haemglobin levels $(p$-value $=0.13)$ and haematocrits $(p$-value $=0.14)$ at four hours after birth. Jaiswal et al, did not exclusively study the effect on term infants delivered by caesarean delivery rather they included new borns delivered by caesareans as well as vaginal delivery. 


\section{Neonatal Apgar Score And Haematocrit}

Rabe et $a l,{ }^{5}$ compared the umbilical cord milking with delayed cord clamping in preterm infants and their results showed no significant difference in haemoglobin levels $(p=71)$ and haematocrit levels $(p=0.65)$ one hour after birth. They did not find a significant difference in mean APGAR score at 5 minutes after birth $(p=.11)$, supplemental oxygen $(p=0.74)$ ventilation requirement $(p=0.85)$. This study results are comparable to Rabe et al, but their study groups included premature infants and they measured haematocrit one hour after birth and need of ventilatory support and supplemental oxygen beyond 24 hours. Investigation into the efficacy and safety of umbilical cord milking in a systematic review by Wassia et al, ${ }^{19}$ showed that umbilical cord milking results in higher haemglobin levels during the neonatal life, with a decreased oxygen requirement, lower the risk of inraventricular bleed without increasing the risk of polycythemia or hyperbilirubinemia. These benefits of umbilical cord milking are comparable to results achieved by delayed cord clamping. Moreover the placental transfusion due to the milking of umbilical cord can decrease the risk of neurological injury by improving systemic circulation in general and decreasing the fluctuations in cerebral perfusion pressures in specific.

\section{LIMITATION OF STUDY}

We did not follow the study population beyond 24 hours so the effect of delayed cord clamping or umbilical cord milking on prevention of early childhood iron deficiency anaemia and better neuro-development can not be predicted in local population.

\section{CONCLUSION}

Milking of the umbilical cord was observed an effective alternative to delayed cord clamping during caesarean surgery. Milking of the umbilical cord is devoid of any adverse effect on haemaglobin, APGAR score or any other physiological disturbance leading to requirement of supplemental oxygen or ventilatory support in first 24 hours. This technique can be of more value when conditions like birth asphyxia demand immediate handing over of the newborn to resuscitation team.

\section{Conflict of Interest: None.}

\section{Authors' Contribution}

GS: Conception, study design, acquisition of data, drafting, final approval, AR: Conception, acquisition of data, revision of draft, final apporval, SM: Analysis of data, revision of draft, final approval, SS: Acquisition of data, revision of draft, final approval, AK: Interpretation of data, revision of final draft, final approval, SI: Conception, review and final approval.

\section{REFERENCES}

1. Li HT, Luo S, Trasande L, Hellerstein S, Kang C, Li JX, et al. Geographic variations and temporal trends in cesarean delivery rates in China, 2008-2014. J Am Med Assoc 2017; 317(1): 69-76.
2. Committee on Obstetric Practice, American College of Obstetric and Gynaecologists. Committee Opinion no.684: Delayed umbilical cord clamping after birth. Obstet Gynecol 2017; 129(1): e5-e10.

3. Boere I, Smith M, Roest AAW, Te Pas AB. Current practice of cord clamping in the Netherland: a questionnaire study. Neonatol 2014; 107(1): 50-55.

4. Hutchon DJR. Why do obstetricians and midwives still rush to clamp the cord. Br Med J 2010; 341(7784): 1220-1225.

5. Rabe H, Jewison A, Alvarez RF, Crook D, Stilton D. Brighton perinatal study group. Milking compared with delayed cord clamping to increase placental transfusion in preterm neonates: a randomized controlled trial. Obstet gynecol 2011; 117(2 pt 1): 205-211.

6. Ghavam S, Batra D, Mercer J, Kugelman A, Hosono S. Effects of placental transfusion in extremely low birthweight infants: metaanalysis of long- and short-term outcomes. Transf 2014; 54(4): 11921198.

7. Li HT, Trasande L, Zhu LP, Ye RW, Zhou YB. Association of caesarean delivery with anemia in infants and children in 2 large longitudinal Chinese birth cohorts Am J Clin Nutr 2015; 101(3): 523-529.

8. Doom JR. Striking While the iron is hot: understanding the Biological and Neurodevelopmental Effects of iron deficiency to optimize intervention in early childhood. Curr Pediatr Rep 2014; 2(4): 291-298.

9. Katheria AC, Truong G, Cousins L, Oshiro B, Finer NN. Umbilical cord milking versus delayed cord clamping in preterm infants. Paediatr 2015; 136(1): 61-69.

10. Wassia HA, Shah PS. Efficacy and safety of umblica cord milking at birth: a systematic review and meta-analysis. JAMA Pediat 2015; 169(1): 18-25.

11. Chantry CJ, Blanton A, Taché V, Finta L, Tancredi D. Delayed cord clamping during elective cesarean deliveries: results of a pilot safety trial. Matern Health Neonatol Perinat 2018; 4(6): 1-7.

12. Wyllie J, Bruinenberg J, Roehr CC. European resuscitation council guidelines for resuscitation 2015: Section 7. Resuscitation and support of transition of babies at birth. Resuscit 2015; 95(9): $249-63$.

13. Wyckoff MH, Aziz K, Escobedo MB, Kapadia VS, Kattwinkel J, Perlman JM, et al. Part 13: Neonatal Resuscitation: American Heart association guidelines update for cardiopulmonary resuscitation and emergency cardiovascular care. Circul 2015; 132 (Suppl-2): S543-560.

14. Ghirardello S, Di Tommaso M, Fiocchi S, Locatelli A, Perrone B, Pratesi S, et al. Italian recommendations for placental transfusion strategies. Front Pediat 2018; 6(372): 1-16.

15. Katheria A, Rich W, Finer N. Optimizing care of the preterm infant starting in the delivery room. Am J Perinatol 2016; 33(3): 297-304.

16. Tang J, Fullarton R, Samson SL, Chen Y. Delayed cord clamping does not affect umbilical cord blood gas analysis. Arch Gynecol Obstet 2019; 299(3): 719-724.

17. Boere I, Roest AA, Wallace E, ten Harkel AD, Haak MC. Umbilical blood flow pattren directly after birth before delayed cord clamping. Arch Dis Child Fetal Neonatal Ed 2015; 100(2): F121-125.

18. Ashish KC, Målqvist M, Rana N, Ranneberg LJ. Effect of timing of umbilical cord clamping on anaemia at 8 and 12 months and later neurodevelopment in late pre-term andterm infants; a facilitybased, randomized-controlled trial in Nepal. BMC Pediatr 2016; 16(35): 1-6.

19. Andersson $O$, Lindquist $B$, Lindgren $M$, sternqvist $K$, Domellof $M$, Hellstrom-Westas L. Effect of delayed cord clamping on neurodevelopment at 4 years of age:a randomized clinical trial. JAMA Paediatr 2015; 169(7): 631-638.

20. Pisacane A. Neonatal prevention of iron deficiency. Br Med J 1996; 312(7024): 136-137.

21. Chaparro CM, Neufeld LM, Alavez GT, Cedillo REL. Effect of timing of umbilical cord clamping on iron status in maxican infants: a randomized controlled trial. Lancet 2006; 367 (9527): 1997-2004.

22. Jaiswal P, Upadhayay A, Gothwal S, singh D, Dubey K, Garg A, et al. Comparison od two types of intervention to enhance placental redistribution in term infants:randomized control trial. Eur J Pediatr 2015; 174(9): 1159-1167. 